\title{
Testing models of rotating stars
}

\author{
Adrian T. Potter and Christopher A. Tout \\ Institute of Astronomy, The Observatories, Madingley Road, Cambridge CB3 0HA, England \\ email: apotter@ast.cam.ac.uk
}

\begin{abstract}
The effects of rapid rotation on stellar evolution can be profound but we are only now starting to gather the data necessary to adequately determine the validity of the many proposed models of rotating stars. Some aspects of stellar rotation, particularly the treatment of angular momentum transport within convective zones, still remain very poorly explored. Distinguishing between different models is made difficult by the typically large number of free parameters in models compared with the amount of available data. This also makes it difficult to determine whether increasing the complexity of a model actually results in a better reflection of reality. We present a new code to straightforwardly compare different rotating stellar models using otherwise identical input physics. We use it to compare several models with different treatments for the transport of angular momentum within convective zones.
\end{abstract}

Keywords. stars: evolution, stars: general, stars: rotation

\section{Introduction}

It has been known for many years that rapid rotation can cause significant changes in the evolution of stars. Not only does it cause a broadening of the main sequence in the HR diagram but it also produces enrichment of a number of different elements at the stellar surface (Hunter et al. 2009). With new large scale surveys such as VLT-FLAMES now reaching maturity, the amount of data available for rotating stars is growing rapidly (e.g. Evans et al. 2005, 2006). Any rotating stellar model must be able to match the observed changes in chemical enrichment and structure. The treatment of rotation and its induced chemical mixing in stars has changed dramatically over the past two decades. The model of Zahn (1992) has formed the basis for much of the work and many variations from the original model have been used during this time to generate different sets of stellar evolution tracks (e.g. Talon et al. 1997; Meynet \& Maeder 2000; Maeder 2003). There are alternate formalisms (e.g. Heger et al. 2000) that treat the physical processes very differently though no direct comparison of the predictions of each model using the same stellar evolution code has been made. Particular emphasis is often placed on the treatment of meridional circulation. Whereas those models based on Zahn (1992) treat meridional circulation as an advective process, Heger et al. (2000) treat it as a diffusive process. This may lead to significantly different predictions for each model even though both treatments are frequently quoted in the literature for their predictions of the effect of rotation on stellar evolution.

One of the most poorly explored features of stellar rotation models is the treatment of angular momentum transport within convective zones. All current 1D models appear to treat convective zones as a rotating solid body. This isn't necessarily correct and there are strong reasons to explore alternatives such as uniform specific angular momentum. This potentially has dramatic consequences for the evolution because a star with uniform specific angular momentum through its convective core has much more total angular momentum for a given surface velocity. Uniform specific angular momentum also results 
in a strong shear layer at a convective boundary that can drive additional transport of chemical elements.

Now that the amount of data is increasing rapidly because of large scale surveys such as VLT-FLAMES it is important to make a direct comparison of the different models for stellar rotation on a common platform with otherwise identical input physics. Using the Cambridge stellar evolution code (Eggleton 1971; Pols et al. 1995; Stancliffe \& Eldridge 2009) we have produced a code that is able to model rotating stars in 1D under the shellular rotation hypothesis of Zahn (1992). The code, RoSE (Rotating Stellar Evolution), can be easily programmed to run with different models for stellar rotation and can model convective zones under a variety of different assumptions. This allows us to compare a variety of models for stellar rotation and determine what, if any, observable traits could be used to distinguish between them. We foresee two possibilities; either there will be clear observational tests to eliminate certain models or the models will show no testable difference in which case a simplified model could be formulated to produce identical evolutionary predictions.

\section{Physics of rotating stars}

There are a number of physical effects due to rotation that must be taken into account to form a complete model. The first is the distortion to the geometry and change in effective gravity because of the centrifugal force. By suitable averaging over isobars it is possible to evaluate the effect of the distortion of the star on the equations of hydrostatic and radiative equilibrium. The modified equations are

$$
\frac{d P}{d m}=-\frac{G m}{4 \pi r^{4}} f_{p}
$$

and

$$
\frac{d \ln T}{d \ln P}=\frac{3 \kappa P L}{16 \pi a c m G T^{4}} \frac{f_{T}}{f_{p}}
$$

where $P$ is the pressure, $T$ is the temperature, $L$ is the luminosity, $\kappa$ is the opacity, $a$ is the radiation constant, $c$ is the speed of light and $G$ is the gravitational constant. $f_{p}$ and $f_{T}$ are typically of order unity and vary with angular velocity. For non-rotating stars $r$ and $m(r)$ are the radius from the centre and mass inside $r$. In the rotating case their exact definitions are modified sligtly due to distortion of the star because of the centrifugal force. For the full derivation see Endal \& Sofia (1976). Under this framework Endal \& Sofia (1976) also derive an expression for the effective gravity modified by the action of the centrifugal force. For moderate rotation rates the distortion to the star is small. It becomes large only when the rotation rate approaches critical (Tassoul 1978).

Von Zeipel (1924) showed that the thermal flux through a point in a star is proportional to the effective gravity. Because the centrifugal force is stronger at the equator than at the poles, the effective gravity depends heavily on the co-latitude and hence so does the thermal flux. This leads to a violation of thermal equilibrium along isobars and gives rise to meridional circulation. The presence of such a circulation has been considered for a long time and is commonly approximated by Eddington-Sweet circulation (Sweet 1950). Zahn (1992) proposed an alternative but similar treatment of the meridional circulation based on energy conservation along isobars.

Differential rotation is expected to arise in stars because of hydrostatic structural evolution and meridional circulation. Stars are therefore subject to a number of hydrodynamic shear instabilities. The proposed hypothesis by Zahn (1992) is that because of the strong stratification present in massive stars, the turbulent mixing caused by these 
instabilities is much stronger horizontally than vertically. This leads to a situation where the angular velocity variations along isobars are negligible compared to vertical variations. This is referred to as shellular rotation where we describe the angular velocity profile by $\Omega=\Omega(r)$.

In $1 \mathrm{D}$ evolution models convective zones are currently assumed to be in solid body rotation. This may be caused by strong magnetic fields induced by dynamo action (Spruit 1999) but there is no conclusive evidence why this must be the case. Certainly in the Sun we see latitudinal variations in the angular velocity throughout the outer convective layer (Schou et al. 1998). Standard mixing length theory suggests that a rising fluid parcel should conserve its angular momentum before mixing it with the surrounding material after rising a certain distance. This would lead to uniform specific angular momentum rather than solid body rotation. This is supported by a recent MLT-based closure model for rotating stars (Lesaffre et al. 2010) and by 3D hydrodynamic simulations (Arnett \& Maekin 2009). In reality magnetic fields are likely to play some part in the transport of angular momentum but it is uncertain whether these are strong enough to enforce solid body rotation. The asymptotic behaviour of the rotation profile in convective zones could have a profound effect on the evolution of the star, first because the total angular momentum content of a star for a given surface rotation increases dramatically for uniform specific angular momentum and secondly because uniform angular momentum in the convective zone produces a layer of strong shear at the boundary with the radiative zone which drives additional chemical mixing. To explore the different possible behaviours we have introduced, in RoSE, the capacity to vary the distribution of angular momentum in convective zones.

With all of these effects and a suitable spherical average, as described by Zahn (1992), the angular velocity evolves according to

$$
\frac{\partial\left(r^{2} \Omega\right)}{\partial t}=\frac{1}{5 r^{2}} \frac{\partial\left(r^{4} \Omega U\right)}{\partial r}+\frac{1}{r^{2}} \frac{\partial}{\partial r}\left(D_{\text {shear }} r^{4} \frac{\partial \Omega}{\partial r}\right)+\frac{1}{r^{2}} \frac{\partial}{\partial r}\left(D_{\text {conv }} r^{(2+n)} \frac{\partial r^{(2-n)} \Omega}{\partial r}\right)
$$

and the chemical abundances evolve according to

$$
\frac{\partial c_{i}}{\partial t}=\frac{1}{r^{2}} \frac{\partial}{\partial r}\left(\left(D_{\text {shear }}+D_{\text {eff }}+D_{\Omega=0}\right) r^{2} \frac{\partial c_{i}}{\partial r}\right)
$$

where $\Omega$ is the angular velocity, $U(r)\left(3 \cos ^{2} \theta-1\right) / 2$ is the radial component of the meridional circulation, $c_{i}$ is the mass fraction of element $i$ and $n$ determines the distribution of specific angular momentum in convective zones. $D_{\text {conv }}, D_{\text {shear }}$ and $D_{\text {eff }}$ are the various diffusion coefficients describing the various effects of rotation. $D_{\Omega=0}$ is the diffusion coefficient for chemical mixing derived for the non-rotating case. $D_{\text {conv }}$ is non-zero only in convective zones and $D_{\text {shear }}$ and $D_{\text {eff }}$ are non-zero only in radiative zones. The major difference between different models (e.g. Talon et al. 1997; Heger et al. 2000; Meynet \& Maeder 2000; Maeder 2003) is the treatment of the meridional circulation, $U$, the diffusion coefficients, $D_{\text {shear }}, D_{\text {eff }}$ and $D_{\text {conv }}$, and the steady power law distribution of angular momentum in convective zones determined by $n$.

With all of these effects, RoSE is capable of producing stellar evolution tracks for various different models very quickly and it can be readily adapted to include additional effects. On a single $2.83 \mathrm{GHz}$ processor we have produced a grid of stellar evolution tracks between the ZAMS and base of the giant branch for 15 masses and 10 rotation rates in $22 \mathrm{hr}$. More rapid computation may be acheived by sacrificing some numerical accuracy. 




Figure 1. Stellar evolution tracks for stars between $4 M_{\odot}$ and $100 M_{\odot}$ calculated with $R o S E$. Tracks for the 4 different models are almost indistinguishable in the HR diagram.

\section{Results}

For our preliminary results we have run grids for four different rotating models. The grid contained the following masses and initial equatorial rotation velocities,

$$
M / \mathrm{M}_{\odot}=\{4,5,6,8,10,12,15,20,30,40,50,60,80,100\}
$$

and

$$
v_{\text {ini }} / \mathrm{km} \mathrm{s}^{-1}=\{0,20,50,100,150,200,250,300,400,500\} .
$$

For the four different models we used the format of equations (2.3) and (2.4) and the

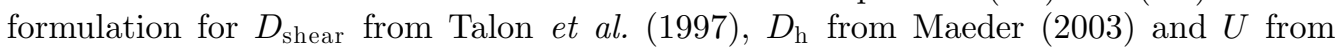
Meynet \& Maeder (2000). We then use the additional parameters listed below.

Model 1: $n=2$ and $D_{\text {conv }}=D_{\mathrm{mlt}}$

Model 2: $n=0$ and $D_{\text {conv }}=10^{16} \mathrm{~cm}^{2} \mathrm{~s}^{-1}$

Model 3: $n=0$ and $D_{\text {conv }}=10^{12} \mathrm{~cm}^{2} \mathrm{~s}^{-1}$

Model 4: $n=0$ and $D_{\text {conv }}=D_{\text {lctp }}$

We derive $D_{\text {mlt }}$ from the typical turbulent velocity and time scales of mixing length theory and $D_{\text {lctp }}$ is from Lesaffre et al. (2010). In the non-rotating limit $D_{\text {lctp }} \rightarrow D_{\text {mlt }}$ $\approx 10^{16} \mathrm{~cm}^{2} \mathrm{~s}^{-1}$. Traditionally, the HR diagram has been the testing ground for variations in stellar models. However, in this case, the four different models predict almost indistinguishible tracks in the HR diagram with variation only at the most massive, most rapidly rotating stars. Even then, the change is small. This suggests that the evolution tracks for rotating stars in the HR diagram are affected almost entirely by the evolution of the angular momentum distribution in radiative zones and not convective zones. We have plotted some of the tracks predicted by the models in figure 1 .

There are a number of additional observational tests to distinguish between different stellar models. One of the most favoured is to look at the nitrogen enrichment produced by rotation by means of a Hunter diagram (Hunter et al. 2009) as shown in figure 2. 

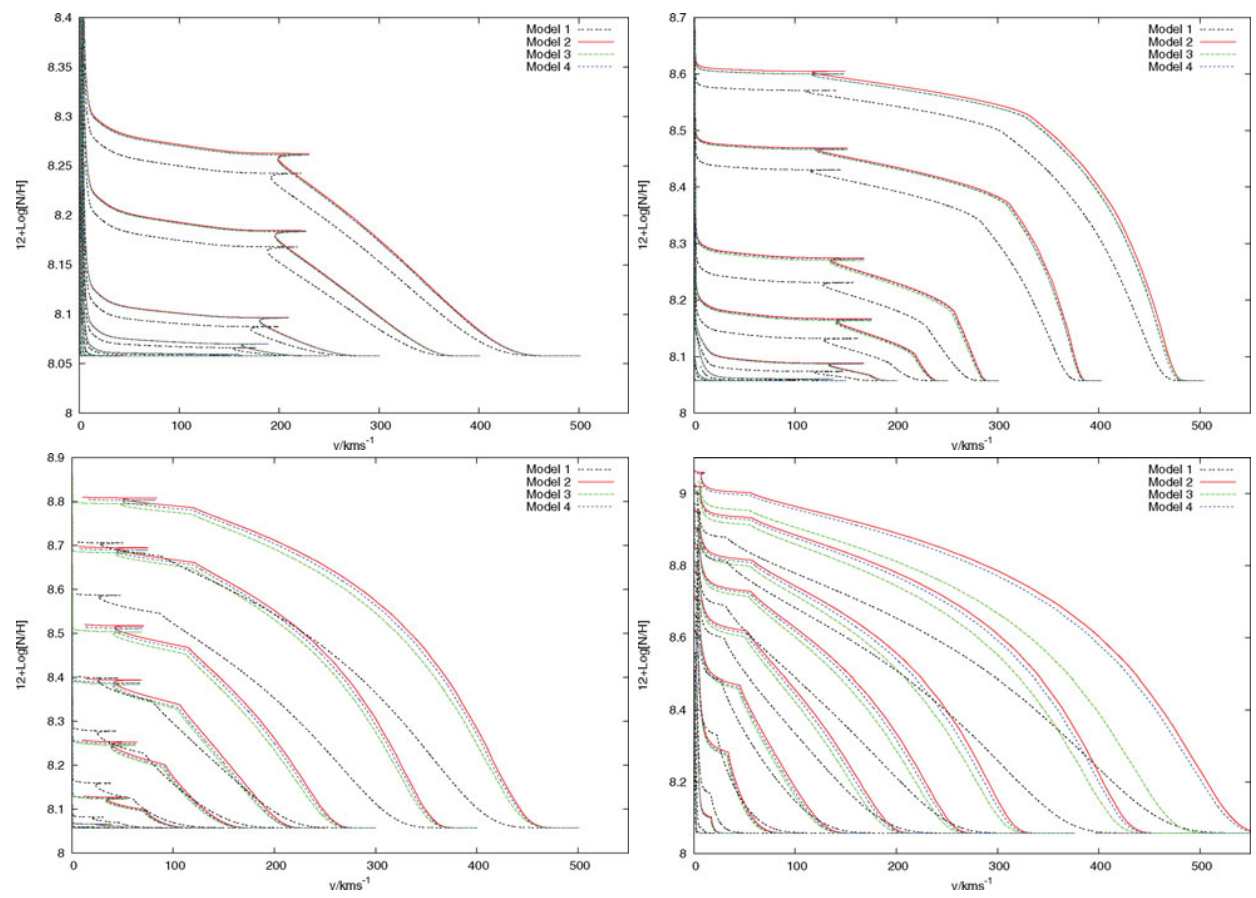

Figure 2. Nitrogen enrichment with a range of initial rotation rates for the 4 different models. Top left is for $10 M_{\odot}$, top right for $20 M_{\odot}$, bottom left for $40 M_{\odot}$ and bottom right for $60 M_{\odot}$. As expected the enrichment is much greater for more massive stars. There is much more enrichment for models in which the core has uniform specific angular momentum, an effect that becomes progressively more pronounced for higher masses.

When we do this we see there are very clear differences between the predictions of each model. Models 2, 3 and 4 which have uniform specific angular momentum throughout their convective zones have significantly more enrichment for all masses and rotation rates but with more pronounced differences for higher mass rapid rotators. However, we note that it is far more difficult to distinguish between these three models with $n=0$. They are almost indistinguishable for masses below $20 M_{\odot}$ and the difference is small even for the $40 M_{\odot}$ model. For the highest mass stars we do see more enrichment in the stars with higher $D_{\text {conv }}$ but $D_{\text {conv }}$ is four orders of magnitude lower in model 3 than model 2. Therefore slighter changes in the diffusion coefficient are unlikely to be dectable by this analysis as indicated by the lack of any signigifcant difference between the results of models 2 and 4 .

\section{Conclusion}

There are many different models currently used to make predicitions regarding the change to stellar evolution as a result of rotation. These models often use very different physics and there are certain aspects of the models, such as angular momentum transport by convection, that remain almost totally unexplored. Now that the available data on rotating stars is starting to increase rapidly, thanks to large scale surveys such as VLTFLAMES, it is important to compare different models and the data to determine whether we can narrow down the number of possible models. Furthermore, by comparing the predictions of the different models, we can provide observational tests that will allow us to eliminate more of the potential models. 
We have shown that, if convection results in uniform specific angular momentum, there is significantly more enrichment in the surface nitrogen abundance of the star compared to similar models where the core is kept in solid body rotation. This does not result in any distinct change in the HR diagram for any rotation rate and masses between $3 M_{\odot}$ and $100 M_{\odot}$ suggesting that evolution tracks in the HR diagram are influenced by the evolution of angular velocity in radiative zones but not convective zones. The amount of enrichment for models with uniform angular momentum in convective zones is only weakly dependent on the diffusion coefficient and changes between the results with $D_{\text {conv }}=10^{12} \mathrm{~cm}^{2} \mathrm{~s}^{-1}$ and $D_{\text {conv }}=10^{16} \mathrm{~cm}^{2} \mathrm{~s}^{-1}$ become significant only for masses above about $60 M_{\odot}$. By calculating the outcome of these models using our new stellar rotation code, RosE, we can be sure that the variations in the models come exclusively from the differences in the model for rotation and not from the numerical method or differences in the rest of the stellar evolution package.

In the future we plan to compute additional models to provide other observational tests to distinguish between them. It will also be important to use the predicitions of each model in detailed population syntheses such as the one by Izzard et al. (2009) in order to suitably compare the predicitions with the data.

\section{Acknowledgements}

ATP thanks the STFC for his studentship and CAT thanks Churchill college for his fellowship.

\section{References}

Arnett, W. D. \& Meakin, C. 2009, in: K. Cunha, M. Spite, \& B. Barbuy (eds.), Chemical Abundances in the Universe: Connecting First Stars to Planets, IAU Symposium 265, p. 106 Eggleton, P. P. 1971, MNRAS, 151, 351

Endal, A. S. \& Sofia, S. 1976, ApJ, 210, 184

Evans, C. J., Smartt, S. J., Lee, J.-K., Lennon, D. J. et al. 2005, A\&A, 437, 467

Evans, C. J., Lennon, D. J., Smartt, S. J., \& Trundle, C. 2006, A\&\&A, 456, 623

Heger, A., Langer, N., \& Woosley, S. E. 2000, ApJ, 528, 368

Hunter, I., Brott, I., Langer, N., Lennon, D. J. et al. 2009, A\& A, 496, 841

Izzard, R. G., Glebbeek, E., Stancliffe, R. J., \& Pols, O. R. 2009, A\&广A, 508, 1359

Lesaffre P., Chitre S. M., Tout C. A., \& Potter A. T., 2010, private communication

Maeder, A. 2003, $A \mathscr{\varepsilon} A, 399,263$

Meynet, G. \& Maeder, A. 2000, A\& A, 361, 101

Pols, O. R., Tout, C. A., Eggleton, P. P., \& Han, Z. 1995, MNRAS, 274, 964

Schou, J., Antia, H. M., Basu, S., Bogart, R. S. et al. 1998, ApJ, 505, 390

Spruit, H. C. 1999, A\&SA, 349, 189

Stancliffe, R. J., \& Eldridge, J. J. 2009, MNRAS, 396, 1699

Sweet, P. A. 1950, MNRAS, 110, 548

Talon, S., Zahn, J.-P., Maeder, A., \& Meynet, G. 1997, A\&\&A 322, 209

Tassoul J.-L. 1978, Theory of rotating stars, Princeton Series in Astrophysics, Princeton: University Press

von Zeipel, H. 1924, MNRAS, 84, 665

Zahn, J.-P. 1992, A\&A, 265, 115 